\title{
Correlation of Brain Weight with Cadaveric Weight, Length, and Cause of Death
}

\author{
Pritija R. Vedpathak ${ }^{1}$, Sadanand S. Bhise ${ }^{2}$, Sudhir D. Nanandkar ${ }^{3}$ \\ ${ }^{1} \mathrm{SDH}$, Mangaon, Dist. Raigad, Maharashtra, India \\ ${ }^{2}$ Professor and Head, FMT, Dr. VMGMC Solapur, OSD DMER and Hoffkin Inst., Mumbai, Maharashtra, India \\ ${ }^{3}$ Dean, Professor FMT, Govt Medical College, Miraj, Maharashtra, India
}

\begin{abstract}
Individual patterns of growth vary widely because of differences in heredity and environment.

Introduction: Human growth from infancy to maturity involves great body size changes and appearance, including the development of sexual characteristics. The growth process is not a steady one; at some times, growth occurs rapidly, at others slowly.

Material and Method: Present study is the prospective examination of a total of 300 autopsy cases brought during the period from 2016-18. In this, 241 were males, and 59 were females.

Result: The data of internal organ weights were statistically analyzed. They are grouped into gender, cadaveric body weight, and cadaveric body length. The cause of death is further grouped into natural and unnatural causes leading to death. Unnatural deaths are grouped into traumatic, asphyxia, and poisoning deaths. Data is analyzed by determining mean, standard deviation, maximum, and minimum.

Conclusion: Brain weight is linearly related to cadaveric weight and cadaveric length. Internal organ weights are linearly related to cadaveric weight and cadaveric length.

Keywords: Autopsy, Organ weight, Bodyweight, etc

Int J Eth Trauma Victimology (2020). DOI: 10.18099/ijetv.v6i02.3
\end{abstract}

\section{INTRODUCTION}

I ndividual patterns of growth vary widely because of differences in heredity and environment. Human growth from infancy to maturity involves great body size changes and appearance, including the development of sexual characteristics. The growth process is not a steady one; at some times, growth occurs rapidly, at others slowly. Weighing of organs at autopsy is not merely an exercise. Still, it has great medico-legal significance, as any deviation from normal range suggests some pathological change in the organ and thus helps in the interpretation of opinion regarding the cause of death and also in finding out the relationship between trauma and death. Children tend to have physiques similar to those of their parents or of earlier forebears; however, the environment may modify this tendency. ${ }^{1}$ Susan Sprogoe-Jackobsen and Ulriksprogoe-Jakobsen et al. (1997) found that there is a positive correlation between body weight and body length in males, but not in females. ${ }^{2}$

The purpose of this study is to provide a practical collection of reference data on a variety of physical measurements like body weight, body length, and internal organ weights for its correlation with the cause of death in different population groups. The present study is a prospective examination of a total of 300 autopsy cases brought during the period from 2016-18 in the mortuary center attached to Forensic Medicine and Toxicology, Tertiary care Medical College and Hospital, Mumbai.
Corresponding Author: Sadanand Sangram Bhise, Professor \& Head, FMT dept, Dr. VMGMC Solapur, OSD DMER and Hoffkin Inst., Mumbai, Maharashtra, India, e-mail: sadanand.bhise@gmail.com

How to cite this article: Vedpathak PR, Bhise SS, Nanandkar $\mathrm{SD}$. Correlation of Brain weight with cadaveric weight, length, and cause of death. Int J Eth Trauma Victimology 2020;6(2):10-13.

Source of support: Nil

Conflict of interest: None declared

Received: 10/07/2020; $\quad$ Received in revised form: 25/09/2020;

Accepted:29/09/2020;

Published:25/12/2020

Human growth from infancy to maturity involves great body size changes and appearance, including the development of sexual characteristics. The growth process is not a steady one; at some times, growth occurs rapidly, at others slowly. Individual patterns of growth vary widely because of differences in heredity and environment. Children tend to have physiques similar to those of their parents or of earlier forebears; however, the environment may modify this tendency. ${ }^{1}$ Susan Sprogoe-Jackobsen and Ulriksprogoe-Jakobsen et al. (1997) found that there is a positive correlation between body weight and body length in males, but not in females. ${ }^{2}$ JB Mukharjee (2007) and JP Modi (1994) found a positive correlation between body weight and organ weight. ${ }^{3,4}$ Gharpure PV and Jhala HI (1949-1958), in their study in Mumbai region found that lower heart weight in persons with lower body weight and weight of organs obtained in this study were lower than 
European studies. In their opinion, neither malnutrition nor low socioeconomic status was the justifiable explanation. They proposed biological, genetic, environmental factors for the same. They also explained higher renal weight (199.45 gms for both kidneys) on the basis of plethoric constitutions, needs of heat loss, the water intake and other habits and conditions in Indians. ${ }^{5-7}$

\section{Materials and Methods}

The present study is a prospective examination of a total of 300 autopsy cases brought during the period from 2016-18. In this 241 were males, and 59 were females. All cases of natural and unnatural deaths reported to the mortuary were considered in this study. The data of internal organ weights were statistically analyzed. They are grouped into gender, cadaveric body weight and cadaveric body length. Data is analyzed by determining mean, standard deviation, maximum and minimum. After that, Pearson's coefficient was used to analyze the relationship between body weight and internal organ weight; body length and internal organ weight with statistical significance at $\mathrm{p}<0.05$. Simple linear regression was performed using the fitted equation $\left(\mathrm{Y}=\mathrm{a}+\mathrm{bx}_{1}+\mathrm{cx}_{2}+\mathrm{dx}_{3}\right.$ ) between the weight of internal organs (dependant) and body weight, body length, age and sex (independent) variables. The internal organ weight, bodyweight, bodylength, and sex together with age were correlated, and so is the reason to calculate them for simple linear regression in the present study.

\section{RESULTS}

The data of internal organ weights were statistically analyzed. They are grouped into gender, cadaveric body weight, and cadaveric body length. The cause of death is further grouped into natural and unnatural causes leading to death. Unnatural deaths are grouped into traumatic, asphyxia, and poisoning deaths. Data is analyzed by determining mean, standard deviation, maximum and minimum. The results are plotted in graphs to find out the trend and predict the relationship of these variables. After that, Pearson's coefficient was used to analyze the relationship between body weight and internal organ weight; body length and internal organ weight with statistical significance at $\mathrm{p}<0.05$. Simple linear regression was performed using the fitted equation $\left(\mathrm{Y}=\mathrm{a}+\mathrm{bx}_{1}+\mathrm{cx}_{2}+\mathrm{dx}_{3}\right)$ between the weight of internal organs (dependant) and body weight, body length, age, and sex (independent) variables. Y-axis depends upon seven values: a is the "constant" and is the value of $y$ when $x$ is zero; $b, c, d$ is the "slope" of the line, the amount by which the $y$ value increases (or decreases, for negative slope) for each unit of increase in the $\mathrm{x}$ value; $\mathrm{x}$ is the value of $x$ itself. The four variables, including bodyweight, body length, age, and sex were important for analysis. The lowest age was 18 years, and the highest was 70 years.

The ranges of ages are 18-67 in males and 18-70 in females. The mean body weight among males is $61.4 \mathrm{~kg}$ while that in females is $55.6 \mathrm{~kg}$. The body length among male is $160.6 \mathrm{~cm}$, while that in a female is found as $155.8 \mathrm{~cm}$. Of the 300 autopsies performed in the study, $182(60.7 \%)$ were having normal body mass index (BMI), followed by overweight $97(32.3 \%)$, underweight 14(4.7\%), and 07 (2.3\%) were obese (Figure 1).

In Table 1 it is seen that in natural deaths, brain size of 125 males $(79.6 \%)$ and 37 females $(86.0 \%)$ was seen as normal, while 21 males $(13.4 \%)$ and 2 females (4.7\%) showed increased brain size and 11 males (7\%) and 4 females (9.3\%) showed decreased brain size.

In unnatural deaths, the brain size of 43 males $(51.2 \%)$ and 11 females $(68.8 \%)$ was seen as normal, while 40 males $(47.6 \%)$ and 5 females $(31.2 \%)$ showed increase in brain size and 1 -male $(1.2 \%)$ and no female $(0 \%)$ showed decrease in brain size.

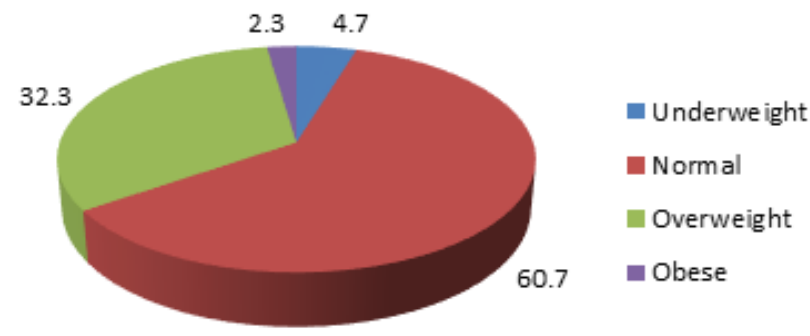

Figure 1: Distribution as per BMI

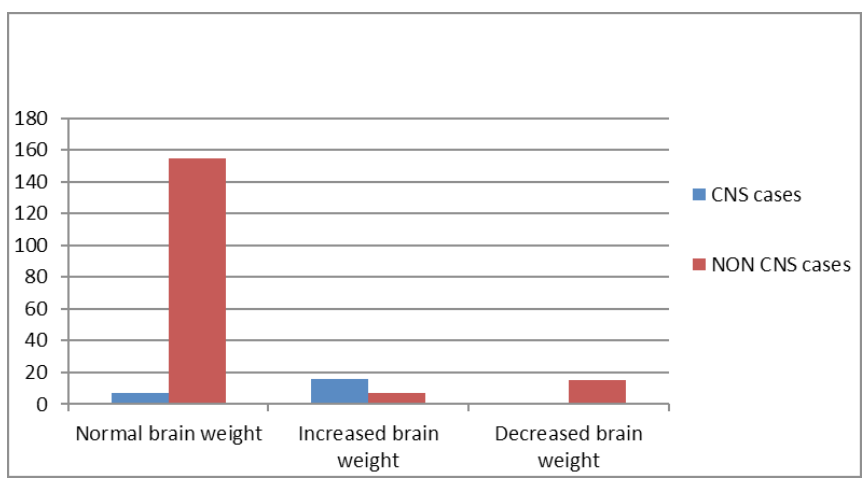

Figure 2: Correlation of brain weight with cause of death

Table 1: Correlation of manner of death with size of the brain

\begin{tabular}{llllll}
\hline & Cause of death & Size & Male (\%) & Female (\%) & Total (\%) \\
\hline Brain & Natural & Normal & $125(79.6)$ & $37(86)$ & $162(81)$ \\
& & Increased & $21(13.4)$ & $2(4.7)$ & $23(11.5)$ \\
& Decreased & $11(7)$ & $4(9.3)$ & $15(7.5)$ \\
& Unnatural & Normal & $43(51.2)$ & $11(68.8)$ & $54(54)$ \\
& Increased & $40(47.6)$ & $5(31.2)$ & $45(45)$ \\
& Decreased & $1(1.2)$ & $0(0)$ & $1(1.0)$ \\
\hline
\end{tabular}


Correlation of Brain weight with cadaveric weight, length, and cause of death

Table 2: Correlation between organ weight and body weight and length of males ( $N=241)$

\begin{tabular}{lllll}
\hline & \multicolumn{3}{l}{ Bodyweight } & Body length \\
\cline { 2 - 5 } Comparison with & $r(p$ value $)$ & interpretation & $r(p$ value $)$ & interpretation \\
\hline BRAIN & $0.263(0.001)$ & positive correlation & $0.095(0.141)$ & Weak positive correlation \\
\hline
\end{tabular}

Table 3: Correlation between organ weight and body weight of females $(n=59)$

\begin{tabular}{lllll}
\hline & \multicolumn{3}{l}{ Body weight } & \multicolumn{2}{l}{ Body length } \\
\cline { 2 - 5 } Comparison with & $r$ (p value $)$ & interpretation & $r(p$ value $)$ & interpretation \\
\hline BRAIN & $0.108(0.413)$ & Weak positive correlation & $0.248(0.058)$ & Weak positive correlation \\
\hline
\end{tabular}

Table 4: Regression equation of the relationship between internal organ weight with body weight, age and sex

\begin{tabular}{lll} 
& Body weight & $R^{2}$ \\
\cline { 2 - 3 } Organ & $\left(Y=a \pm b x_{1} c x_{2} \pm d x_{3}\right)$ & 0.169 \\
\hline Brain & Brain weight $=1145.79+5.25$ (body weight)-3.025(age)-69.64(sex) & \\
\hline
\end{tabular}

Table 5: The norm of internal organ weights (gm) according to males and females.

\begin{tabular}{lll}
\hline \multirow{3}{*}{ Major Organ } & Male $(n=241)$ & Female $(n=59)$ \\
\cline { 2 - 3 } & $($ Mean \pm SD) & (Mean \pm SD) \\
\hline BRAIN & $1270.8 \pm 168$ & $1174.7 \pm 152$ \\
\hline
\end{tabular}

In both natural and unnatural deaths, there is no change of the shape of organ found. In all-natural deaths among males and females, the surface color of the brain was found normal i.e., grey white. While in unnatural deaths, 79 males (94\%) and 16 females $(100 \%)$ show the normal surface color of the brain, and 5 males $(6 \%)$ showed pale white surface color of the brain. In Table 2 it can be seen a positive correlation of brain weight with the Bodyweight of males $(p<0.01)$ and a weak positive correlation with the length of the deceased.

In Tables 3 and 4, it can be seen that a weak positive correlation between brain weight with the body weight and length of females. There is no statistically significant association found. The regression equation of the relationship between internal organ weight with body weight, age, and sex was calculated statistically and found to be "brain weight $=1145.79+5.25$ (bodyweight)-3.025 (age)-69.64 (sex)." The normal brain weight ( Table 5), which was found after analysis of data in males, is 1270.8 Grams with a slandered deviation of 168 grams on either side, and in females, it is 1174.7 grams with slandered deviation of 152 grams on either side.

For estimating the correlation of organ weight with cause of death, the Chi-square test applied for natural causes leading to death in the direction of organ weights with a particular system involved in the cause of death. If we see as per cause of death $3.84 \%$ cases are due to asphyxial death, 41 cases $(64.06 \%)$ deaths are due to trauma to brain, and in poisonrelated cases $3(30 \%)$ cases and in all these cases weight was found to be increased.

\section{Discussion}

The organ weight is a good diagnostic criteria of an autopsy if normality is accurately defined. It is seen that researchers are more interested in weighing organs directly as it is part and parcel of routine post mortem examination. Many conditions like age, sex, cadaveric weight, cadaveric length, nutrition, socioeconomic status, geographic conditions, genetics, habits, diet, diseases etc are known to influence the internal organ weight as well as cadaveric weight and shows great variations.

In a study by Murty OP et al. (2007), ${ }^{8}$ there was a positive correlation between the weight of brain and cadaveric weight, which is consistent with our study. PiyanunMathuramon et al $(2009)^{9}$ observed weight of the brain and heart were slightly increased. ${ }^{9}$ The weight was distributed according to age range, and brain weight was reduced as age increases. This is because under-nutrition in the elderly has a relative effect on brain weight, probably because the brain contains only small amount of glycogen and neutral fat. The weight distributed according to the range of age; brain weight was reduced as age increases. This is because under-nutrition in the elderly has a relative effect on brain weight, probably because the brain contains only a small amount of glycogen and neutral fat. When compared with a study done by Vadgama KD et al. (2014), ${ }^{6}$ they observed a positive correlation between cadaveric weight and the brain's weight in both sexes. Santosh B Bhoi and RizwanAKamle et al. $(2017)^{10}$ observed a positive correlation between the weight of brain with cadaveric weight in both males and females ( $\mathrm{p}$ value $<0.001$ ). ${ }^{10}$ The findings are consistent with our study

Our study shows a weak positive correlation between the weight of the brain and cadaveric length in both males and females; but no statistically significant association was found. When compared with study by Vadgama KD et al. (2014), ${ }^{6}$ there was a negative correlation between weight of brain and cadaver length. Piyanun M et al. (2009) ${ }^{9}$ found relationship between internal organ weight and body weight and body length of males whereas; in females, the weight of internal organs except kidneys were not related to body length. He also found that the weight of male brain had a positive correlation with both body weight and body length. 
Bandyopadhyay Chandan et al. (2017) ${ }^{7}$ observed weight of the brain, in both males and females were positively correlated with cadaveric length, which means that as the body length increases, the weight of the brain under study also increases, but in this study the correlation is of minor degree; while the present study shows a similar result.

Among all natural deaths, the brain size of 125 males $(79.6 \%)$ and 37 females $(86.0 \%)$ was seen as normal, while 21 males (13.4\%) and 2 females (4.7\%) showed increased brain size and 11 males (7\%) and 4 females (9.3\%) showed decreased brain size. In the present study, the surface color of the brain is normal, i.e., grey-white in all deaths due to natural causes.

Among unnatural deaths, size of brain in 43 males (51.2\%) and 11 females $(68.8 \%)$ is seen as normal, while 40 males $(47.6 \%)$ and 5 females $(31.2 \%)$ shows an increase in brain size and 1 -male $(1.2 \%)$ and no female $(0 \%)$ shows decrease in brain size. Increased size of brain is mostly attributed to cerebral edema, which results following acute CNS injury, cells of the neurovascular unit in particular endothelial cells and astrocytes resulting in maladaptive ion transport and generation of abnormal osmotic forces. The brain's surface color in 79 males $(94 \%)$ and 16 females (100\%) is seen as normal, i.e., grey-white. And 5 males $(6 \%)$ showed pale white surface color of the brain, the condition associated with hemorrhagic shock.

\section{Conclusion}

The weight of brain in females is less than in males at all ages. Brain weights is linearly related with cadaveric weight and cadaveric length. Brain weight is directly affected with either increase or decrease of their weights in relation with cause of death, both in natural as well as unnatural deaths. This study can be considered as useful anatomical data to understand the disease property in this regional population. Weight of brain is useful tool to enlighten the pathological course undergone into the body of deceased, hence helping in establishing the cause of death for the better administration of justice in medico-legal cases. This study can be compared and correlated with many similar studies by different autopsy surgeons, which may form a new era regarding morphological study of human organs and its correlation with cause of death.

\section{Recommendations}

The sample size is small in the present study, hence it should be increased to make the data more qualitative and reliable for reference purposes. It will be better if the number of females cadaver cases are increased because they are fewer in number in the present study, thus may affect statistical evaluation.

\section{REFERENCES}

1. JM. Human development process of growth and change. In: Encyclopedia britannica.com/ science. 2016. p. 1.

2. Sprogøe-Jakobsen S, Sprogøe-Jakobsen U. The weight of the normal spleen. Forensic Sci Int. 1997;88(3):215-223.

3. Mukherjee J. Textbook of Forensic Medicine and Toxicology. 3rd ed. West Bengal (India): Academic publishers; 2007. p. 1224-25.

4. Modi J. Textbook of Medical Jurisprudence and Toxicology. 21st ed. Nagpur (India); 1994. p. 332.

5. Gharpure P, Jhala H. Relation of body weight to organ weights. Indian Med Gaz. 1949;84:541.

6. Gharpure P, Jhala H. Relation of body weight to organ weight. Indian Med Gaz. 1950;85(8):342.

7. Gharpure P, Jhala H. Normal standards for body weights and organ weights in India. Indian Med Gaz. 1958;93:445-447.

8. Murty OP, Ling CS, Suhashni G, Mohd Fahmee AR, Nurasma Z, Khalsom S. Organ weights of Malay, Chinese, Indian, and Indonesian ethnic groups in Malaysia. Mal J Path Sci. 2007;2(1):53-83.

9. Mathuramon P, Chirachariyavej T, Peonim AV, Rochanawutanon M. Correlation of internal organ weight with body weight and length in normal Thai adults. Med J Med Assoc Thail. 2009;92(2):250.

10. Bhoi SB, Kamle RA, Meshram SK, Waghmare SA, Shirsat KB. Study of correlation of organ and body weight during autopsy with regard to age and sex in adult population at Solapur region. Indian J Forensic Community Med. 2017;4(2): $115-120$. 